Proceedings

\title{
Comparison of Leading Biosensor Technologies to Measure Endothelial Adhesion, Barrier Properties and Responses to Cytokines in Real-Time ${ }^{+}$
}

\author{
James JW Hucklesby 1,2,*, Akshata Anchan ${ }^{2,3}$, Simon J O'Carroll ${ }^{3,4}$, Catherine E Angel ${ }^{1}$ \\ and E Scott Graham 2,3,* \\ 1 School of Biological Sciences, Faculty of Science, University of Auckland \\ 2 Department of Molecular Medicine and Pathology, Faculty of Medical and Health Sciences, University of \\ Auckland \\ 3 Centre for Brain Research, University of Auckland; s.graham@auckland.ac.nz \\ 4 Department of Anatomy and Medical Imaging, Faculty of Medical and Health Sciences, University of \\ Auckland; s.ocarroll@auckland.ac.nz \\ * Correspondence: james.hucklesby@auckland.ac.nz (J.J.W.H.); s.graham@auckland.ac.nz (E.S.G.) \\ + Presented at the 1st International Electronic Conference on Biosensors, 2-17 November 2020; Available \\ online: https://iecb2020.sciforum.net/.
}

Received: date; Accepted: date; Published: date

\begin{abstract}
Background: ECIS, xCELLigence and cellZscope are commercially available instruments, able to measure the impedance of cellular monolayers continuously and with high precision. The small currents used allow for label-free, real-time monitoring of the cells in a non-invasive manner. Despite the widespread use of these systems individually, direct comparisons between the systems has not been published. To compare the sensitivity of the instruments, responses of the brain microvascular endothelial cell line hCMVEC to the inflammatory cytokines TNF $\alpha$ and IL1 $\beta$ were measured on all three instruments simultaneously. All three instruments showed transient decreases followed by prolonged increases in impedance. Although xCELLigence could detect these changes, it was unable to determine which component of the barrier was affected. In contrast, ECIS and cellZscope were both able to attribute responses to particular barrier components, where ECIS had a higher sensitivity than the cellZscope. Finally, as the cellZscope uses Transwells, it allows access to the basolateral compartment, an important advantage of this technology. Furthermore, although $\times C E L L i g e n c e$ readings are equivalent to ECIS, the reduced frequency range greatly limits interpretation. This work demonstrates that instruments must be carefully selected to ensure they are appropriate for the experimental questions being asked.
\end{abstract}

Keywords: ECIS; xCELLigence; cellZscope; HMVEC; neuroinflamation; impedance sensing

\section{Introduction}

Impedance sensing is a label free, real time technique to monitor cellular function. First pioneered by Giever and Keese, cells can be measured by exposing the cells to very small currents $[1,2]$. By measuring the resistance that the cells provide to this current, we can accurately measure the responses of the cells in real time. As no labeling is required, this is highly non-invasive and can be carried out over extended periods to give high-resolution information over long periods. Furthermore, this information is quantified and hence able to be directly analyzed statistically. Mathematical models can also be applied to this data to allow exploration of various cellular parameters that cannot be readily measured directly [3]. These advantages have triggered broad adoption of impedance sensing in a wide variety of applications, with a range of custom instruments 
having been developed [4-7]. However, adoption of these systems has been limited as constructing a specialist instrumentation is technically challenging. In contrast, commercially available instruments provide a turnkey solution to accessing impedance sensing. In this paper, we compare three such systems, the physical characteristics of which are summarized in Figure 1.

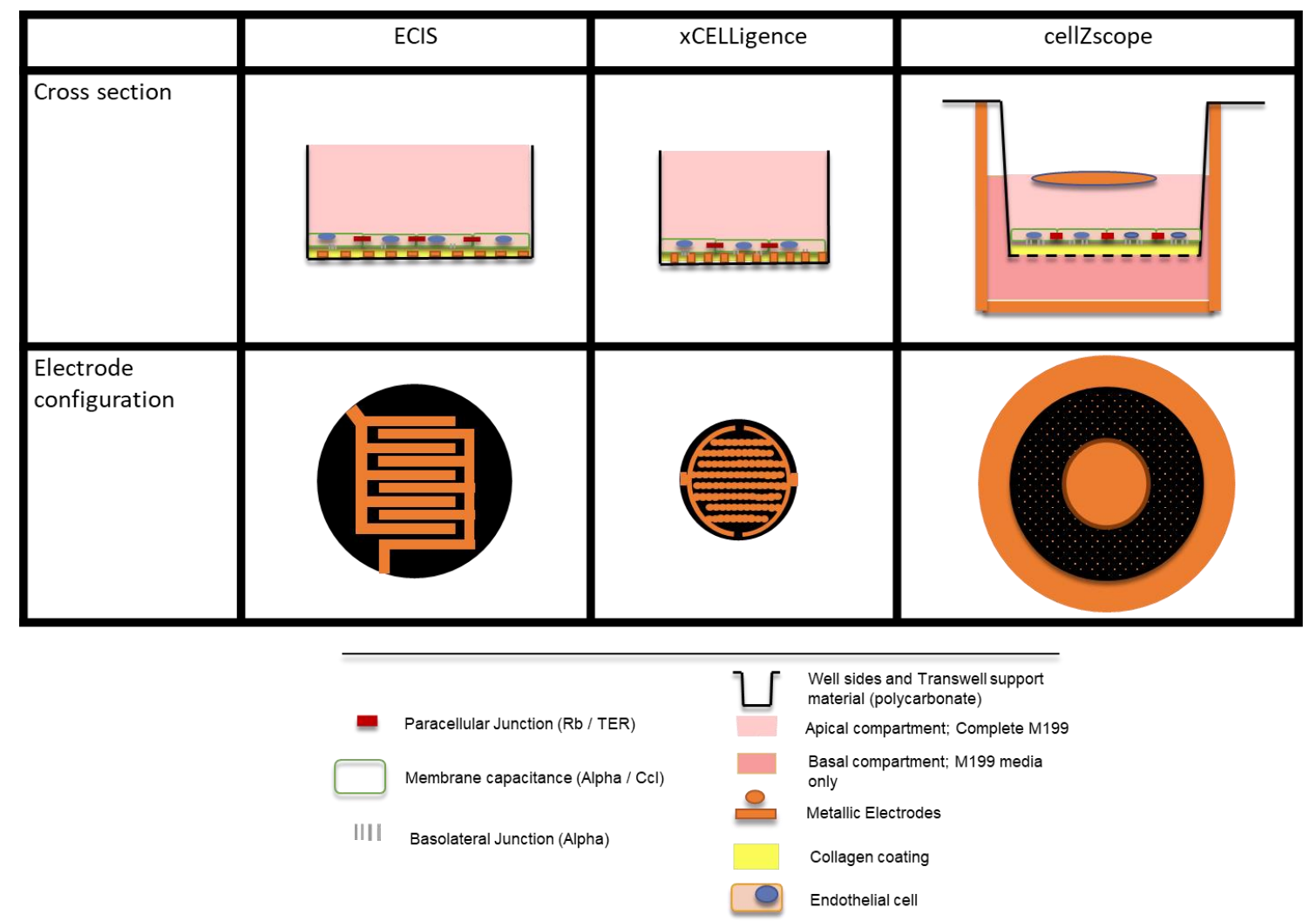

Figure 1. Comparison of commercially available instruments for impedance sensing reveals that the electrode arrays are considerably different in physical configuration.

Giever and Keese's original ECIS invention has since been commercialized by Applied Biophysics as the ECIS Z0 [8]. This instrument uses 96 well plates with electrodes fabricated directly onto the base of the chip. Impedance and phase measurements at a range of frequencies is collected by the instrument, and subsequently these can then be modelled computationally to indicate biologically relevant cellular parameters. This results in three values being generated, $\mathrm{Rb}, \mathrm{Cm}$ and Alpha. $\mathrm{Rb}$ represents the cell-cell interaction between endothelial cells; $\mathrm{Cm}$ represents the membrane resistance of the cells, whilst Alpha represents the distance between the cells and the underlying substrate (basolateral adhesion) [3]. Together, these values allow for in-depth analysis of biological responses [9].

More recently, ACEA Biosciences (now part of Agilent) released the xCELLigence instrument [10]. Much like ECIS, this instrument uses gold electrodes fabricated directly onto the base of a 96 well plate. However, this instrument only collects impedance measurements at three frequencies, making modeling impossible.

Finally, the cellZscope is the most recent addition to the market, and is able to measure impedance across a Transwell filter [11]. The Transwell is seated in a metal pot that acts as an electrical conductor, whilst a second electrode is suspended over the cells, making contact with the media in the apical chamber, completing the circuit and allowing impedance to be measured. Like ECIS, phase and impedance data are collected at a range of frequencies. Because impedance is captured at a number of frequencies, modelling can also be conducted. This results in the calculation of TER as a measurement of cell-cell junctional interaction and $\mathrm{Ccl}$ as a measure of membrane impedance [11]. An equivalent of the Alpha value generated by the ECIS instrument is not included 
in this model, as the porous nature of Transwells means that this measurement is not physically present and therefore not sensible to infer.

Despite the abundance of studies using these instruments, direct comparisons between these instruments have not been conducted. This is a critical lack of knowledge, as the inferences from data collected from all three instruments are regularly used together to interrogate cellular responses [1216]. Therefore, in this paper we use all three commercially available instruments to analyze the similarities and differences between all three instruments. The hCMVEC cell line was chosen due to its low overall resistance, which although characteristic of brain microvascular endothelial cell lines, makes study challenging [17].

The inflammatory cytokines TNFa and IL1 $\beta$ were selected for these experiments due to their well-defined and biphasic response. The response of hCMVECs to IL1 $\beta$ and TNF $\alpha$ has been explored on a molecular level, and the response has been well-characterized using impedance instruments [18]. These responses are ideal for these studies, as the cytokines first cause a decrease in resistance, followed by a substantial increase for an extended period. This change reflects an initial weakening of the paracellular barrier strength followed by a sustained strengthening of the endothelial barrier. These treatments therefore allows decreases and increases in resistance to be examined from the same stimulus. Furthermore, this is an ideal response to use impedance sensing for as the transient nature makes the response difficult to examine with traditional single time point analysis as the initial decrease would be easy to miss [19]. Therefore, a concentration at the high end of the physiological range was selected to give a robust response, with good characteristics for testing the impedance instruments.

We also want to evaluate two key parameters of the data produced, difference in magnitude at key points in time and difference in the shape of the curve. A difference in magnitude is useful as it has a straightforward interpretation, and correlates well with traditional single time point assays $[9,12]$. The second characteristic is a difference in shape of the curve, as even if two responses have the same magnitude at a key time point they may reach that point in a very different way. This was analyzed using cross correlation, which generates a single value between 1 and -1 for each pair of curves. A value of 1 represents identical curves, -1 represents opposite curves and 0 shows no correlation between the two readings [20]. By assessing both these characteristics in concert, we are able to robustly compare the responses from all three systems.

In this paper, we run the same experiment simultaneously on all three impedance-sensing instruments. We then show that although the instruments impedance measurements have similar shapes, they differ in magnitude demonstrating significant differences in sensitivity. Furthermore, the different modeled measurements increase the sensitivity of the measurements significantly, and these values cannot be directly inferred from overall impedance measurements. Overall, this demonstrates the importance of selecting the most appropriate instrument for any particular experiment.

\section{Materials and Methods}

\subsection{Culture of the Brain Endothelial Cells}

Human cerebral microvascular endothelial cells (hCMVECs) were purchased from Applied Biological Materials Inc (cat\# T0259). The cells were cultured in $75 \mathrm{~cm}^{2}$ Nunc flasks (cat\# 156499) using M199 medium containing 10\% FBS, $1 \mu \mathrm{g} / \mathrm{mL}$ hydrocortisone, $3 \mathrm{ng} / \mathrm{mL}$ hFGF, $1 \mathrm{ng} / \mathrm{mL}$ hEGF, $10 \mu \mathrm{g} / \mathrm{mL}$ heparin, $2 \mathrm{mM}$ GlutaMAX and $80 \mu \mathrm{M}$ dibutyryl-cAMP, later referred to as complete M199 medium. For both cell maintenance and experiments, cultureware was were coated with $1 \mu \mathrm{g} / \mathrm{cm}^{2}$ collagen I dissolved in $0.02 \mathrm{M}$ acetic acid at room temperature for $1 \mathrm{~h}$ before being washed 3 times with sterile MilliQ water, prior to cell plating. To passage the cells, T75 flasks were washed twice with $4 \mathrm{ml}$ of prewarmed PBS before being incubated with $4 \mathrm{ml}$ of pre-warmed TrypLE for $5 \mathrm{~min}$. The TrypLE was then neutralized with an additional $4 \mathrm{ml}$ of complete M199 before cells were centrifuged at $100 \times g$ for $5 \mathrm{~min}$, counted and prepared to the appropriate concentration for experimental seeding. All experiments used 
cells between passages 11 and 16 . All impedance instruments and cell cultures were kept in dedicated incubators at $37^{\circ} \mathrm{C}, 5 \% \mathrm{CO}_{2}$ and $100 \%$ humidity.

\subsection{Preparation of Cells in Impedance Sensing Technology}

ECIS: 96W20idf plates were treated with $10 \mathrm{mM}$ cysteine for $15 \mathrm{~min}$ to standardize electrode impedance. The wells were then collagen coated as described above. The hCMVECs were seeded at 80,000 cells per well in $200 \mu \mathrm{L}$ of complete M199 medium. Cells were allowed to proliferate for $48 \mathrm{~h}$, allowing all units to stabilize. The ECIS machine was run continuously in multi-frequency mode using the default frequency spectra.

xCELLigence: E-plates (96 wells) were collagen coated as described above. The plate was then flooded with $122 \mu \mathrm{L}$ of complete M199 and well calibration conducted. The plate was then returned to the hood and the media replaced with $122 \mu \mathrm{L}$ of media containing 48,800 cells per well to account for the decreased surface area of an xCELLigence plate compared to an ECIS plate. Cells were then allowed to proliferate for $48 \mathrm{~h}$ until the Cell Index (CI) had stabilized.

cellZscope: Before the experiment began, all cellZscope components were cleaned with MiliQ water, $70 \%$ ethanol and then MiliQ water again. Pots and dipping electrodes were then autoclaved to ensure sterility. Before coating began, the Cell Module was assembled under sterile conditions and each of the stainless steel pots was flooded with $900 \mu \mathrm{L}$ of basal M199 media. The assembled module was then placed in the cell culture incubator to equilibrate for at least one hour. Transwells (Corning) were coated from the apical side as previously described. The hCMVECs were then seeded into the apical chamber at 80,000 cells per well, in $200 \mu \mathrm{L}$ of complete M199 medium. Transwells were then transferred into the Cell Module, taking care not to trap any bubbles underneath the membrane. The cell module was then placed in the instrument, and spectra were acquired at the highest resolution between 1 and 100K ohms every $15 \mathrm{~min}$.

\subsection{Treatment with Inflammatory Cytokines}

On the day of treatment, complete M199 was pre-warmed, and either TNF $\alpha$, IL1 $\beta$ or an equivalent amount of water (henceforth labeled as control) was added to form a $5 \times$ stock. Each instrument was then paused and the stock gently introduced to the middle of the well or apical chamber to reach the final concentration required. Cells were then returned to the instruments and measurements resumed. Monitoring continued on all instruments for a further $27 \mathrm{~h}$.

\subsection{Data Analysis}

RStudio (version 1.1.414, RStudio, Inc., Boston, MA, USA) and the vascr package developed by James Hucklesby (unpublished) was used to import data and generate cross correlation values. vascr uses the ccf function in the stats package to run the underlying cross correlation analysis. Modeling was completed in the software provided by the vendor for each instrument respectively against a cell-free well in the same experiment.

Graphs: Graphs were generated using ggplot2 version 3.3.2. All graphs show mean \pm sem of the replicates from one representative experiment. All experiments were repeated at least three times, and similar results were observed.

\section{Results and Discussion}

To assess the comparability of the three instruments, we first collected impedance spectra of a confluent monolayer at $47 \mathrm{~h}$ after simultaneous seeding of either 80,000 cells or media only (Figure 2). This time point was selected as the cells had formed a stable monolayer in all units collected by all three instruments for an extended period, providing the baseline for further treatments. As all three instruments were seeded simultaneously using the same preparation of cells, we can directly compare the measurements collected. 


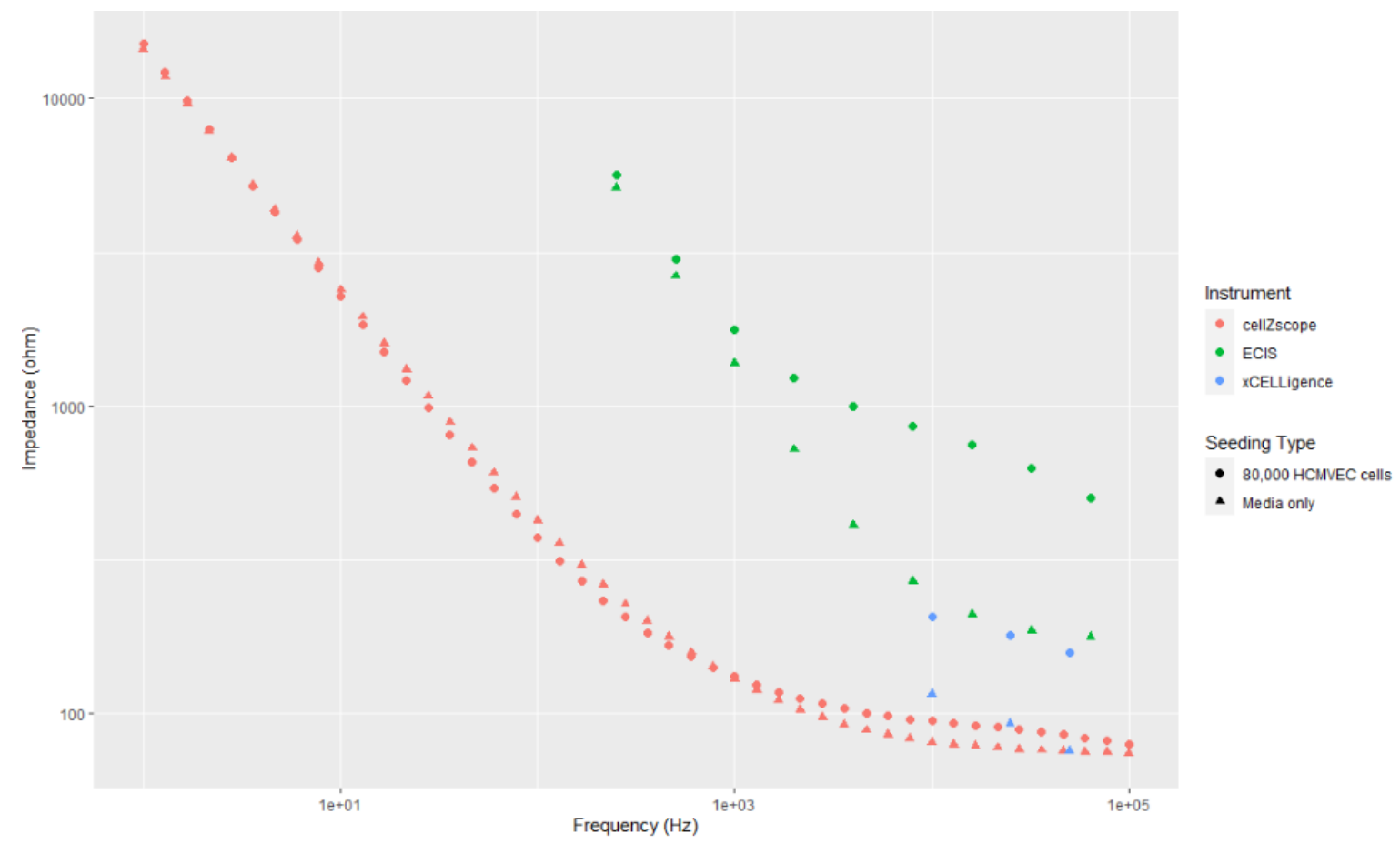

Figure 2. Impedance spectra of HCMVEC cells in different instruments. Either media only or media containing 80,000 cells per well was plated into each instrument and impedance spectra acquired after $47 \mathrm{~h}$. Each dot represents the mean value at each frequency where impedance was measured, with dots representing cell containing wells and triangles representing media only wells.

This dataset immediately demonstrates the quantity of data collected by each instrument, and the relative concordance across all three instruments. The cellZscope captures 34 data points, compared to 9 from the ECIS instrument and only 3 from the xCELLigence. The small number of data points collected over a relatively narrow range makes it clear why the xCELLigence data cannot be modelled, as it is impossible to determine the overall impedance response of the cells. The trends from the ECIS and xCELLigence instruments were similar, showing only a slight sigmoidal shape. This lack of a clear sigmoidal shape, characteristic of cell lines with a low overall impedance, demonstrates the power of these impedance instruments to detect even slight barrier function. However, this difference is not immediately obvious, and therefore modelling (conducted later in this paper) will be invaluable in interpreting these datasets.

Although all three trends are similar and distinct from media only controls, the values are not directly comparable in magnitude. This is due to the drastic differences in electrode area and configuration between instruments, which affect the absolute value of the impedance measured. Despite this, the trends are similar, demonstrating that similar cellular characteristics are being measured. Furthermore, all three instruments were able to detect the cellular monolayer's presence, as all values increased from their media only values. This difference was particularly apparent in the ECIS and xCELLigence instruments where the cells are in direct contact with the electrodes; however, a change in shape was still apparent on the cellZscope. Although this demonstrates that the presence of a static endothelial monolayer can be detected, it does not speak to the ability to discern responses from the cells.

To test the sensitivity of each system to detect cellular responses, the confluent cellular monolayers were treated with the inflammatory cytokines TNF $\alpha$ and IL1 $\beta$ (Figure 3) before impedance readings were continued. To obtain the purest comparison across all instruments frequencies as similar as possible to each other were selected. The data was then normalized to one hour before treatment, and is presented as a percentage change to allow for direct comparison despite the large differences in baseline measurements between instruments. Finally, cross correlation was calculated to test the instrument's ability to separate the curves shapes. 



Figure 3. ECIS, cellZscope and xCELLigence readings are able to detect the responses of hCMVEC cells to inflammatory cytokines. HCMVEC cells were plated at a density of $250,000 \mathrm{cells} / \mathrm{cm}^{2}$ and incubated for $48 \mathrm{~h}$ until confluent. Cells were then treated with either TNF $\alpha$ or IL1 $\beta$, and monitored for a further $48 \mathrm{~h}$. Note the lower the correlation value the more different the response. Data presented is the mean \pm SEM of three wells in a representative experiment normalized to the value of the well at $47 \mathrm{~h}$. Cross correlation shown is calculated between the means of each treatment type.

Although all instruments were able to detect differences between the treatments, ECIS readings were by far the most sensitive. IL1 $\beta$ caused a $9 \%$ reduction in the raw ECIS impedance, followed by an increase of $5 \%$ by the $70 \mathrm{~h}$ time point. Although the cellZscope had a similar increase above vehicle at $70 \mathrm{~h}$, the initial loss of barrier function was much less pronounced. The xCELLigence was also able to detect both the increasing and decreasing impedance, however the change in magnitude of both phases was much less than that of the ECIS machine. The ECIS instrument's ability to resolve absolute differences in both phases also helped generate large differences in cross correlation coefficients between the different treatments. The results were striking, ranging between almost perfect correlation between the two inflammatory stimuli to -0.3 between each inflammatory stimulus and the control well. This demonstrates that ECIS is able to unambiguously distinguish between the stimuli. The other two instruments are still able to make a distinction, but their sensitivity is considerably less.

To achieve further sensitivity, ECIS data was then modeled and the cross-correlation analysis repeated (Figure 4). Although the ECIS was already the most sensitive to magnitude and shape changes, this effect was further amplified when $\mathrm{Rb}$ was examined. Although all three samples had similar shapes and high cross correlation values, the difference in magnitude was immediately evident. Furthermore, the trend in $\mathrm{Cm}$ was very different to that of $\mathrm{Rb}$, and effect was not evident from examination of impedance data alone. This demonstrates the strong ability of ECIS modeling to separate cellular phenomenon, and highlight particular areas for further examination. 
ECIS



$\mathrm{Rb}$
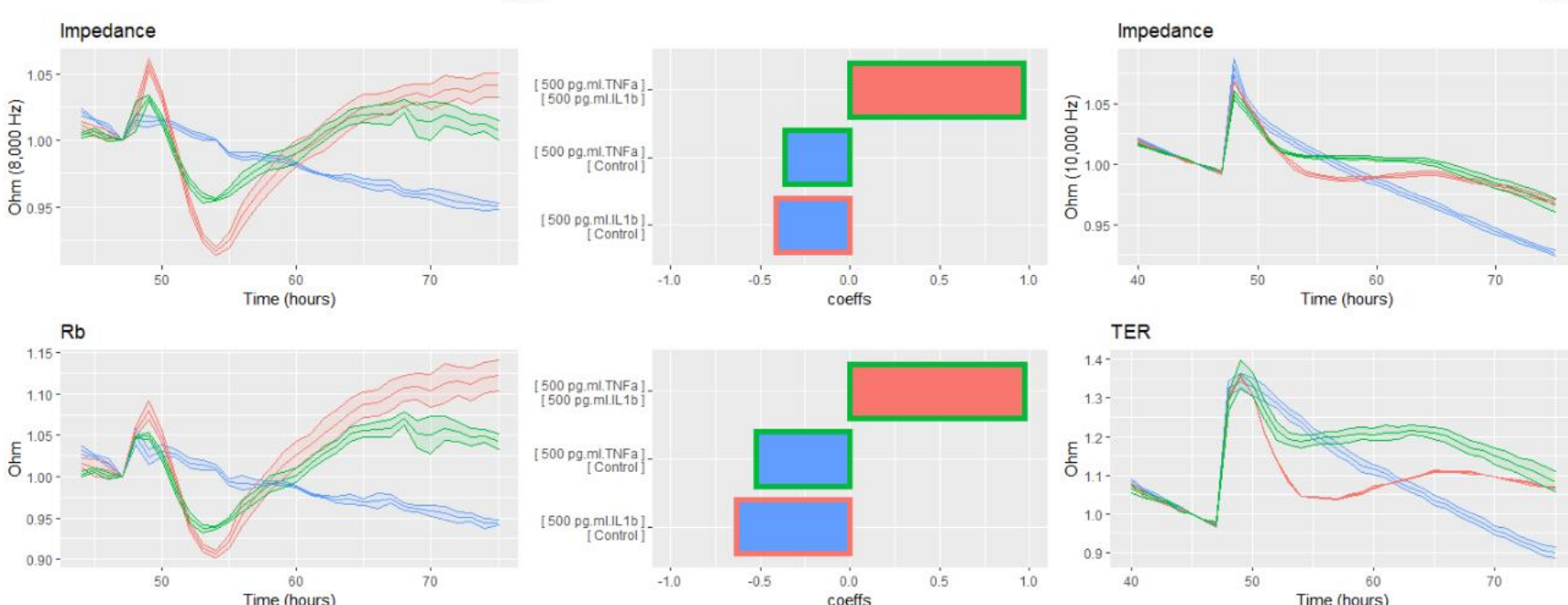

cellZscope


Figure 4. Attribution of impedance to cellular phenomena increases the sensitivity of the instruments to inflammatory treatment. HCMVEC were plated at a density of 250,000 cells $/ \mathrm{cm}^{2}$ and continuously monitored for $48 \mathrm{~h}$. Cells were then treated with TNF $\alpha$, IL1 $\beta$ or vehicle and measurement was continued for a further $48 \mathrm{~h}$. Cross correlation analysis was then carried out between the average curves from all treatments for each modelled unit. Results show the mean \pm SEM from one representative experiment normalized to the value of the well at $47 \mathrm{~h}$. 
Modeling of cellZscope data also increased experimental sensitivity, and allowed for additional inference. Compared to raw impedance, TER amplified the response to both cytokines, lowering the cross correlation and increasing magnitude changes. Whilst the cell-cell association values were similar, with the trend in TER being similar to that that observed in Rb using ECIS; the response in membrane capacitance values was different between the two instruments. Rather than generating a sustained decrease in $\mathrm{Cm}$, the inflammatory response in $\mathrm{Ccl}$ was transient and had a much larger difference in shape between stimuli. This may be due to differences in cellular morphology between those cultured on Transwells versus those grown on solid surfaces. The ability for modeling to increase the sensitivity of cellZscope data also provides opportunities to study less pronounced cellular phenomena, particularly those where basolateral access is required and therefore the more sensitive ECIS instrument is unable to be employed.

Given the significant utility of modelled data, we attempted to correlate xCELLigence data (Figure 5, Purple) with both modelled and raw parameters acquired from ECIS measurements. Although the systems are very similar in their physical setup, the impedance measurements collected were not identical. The cross correlation and magnitude of readings between the two instruments was very high in the control well; however, this similarity was not present in cells treated with inflammatory cytokines. Of all the ECIS units, Cm was the most similar in shape to the xCELLigence readings, although the magnitude was very different. Furthermore, even the relationship between the ECIS units and the xCELLigence measurements was inconsistent. Therefore, it is impossible to use xCELLigence as a proxy for any particular measurement, and it must be interpreted independently.
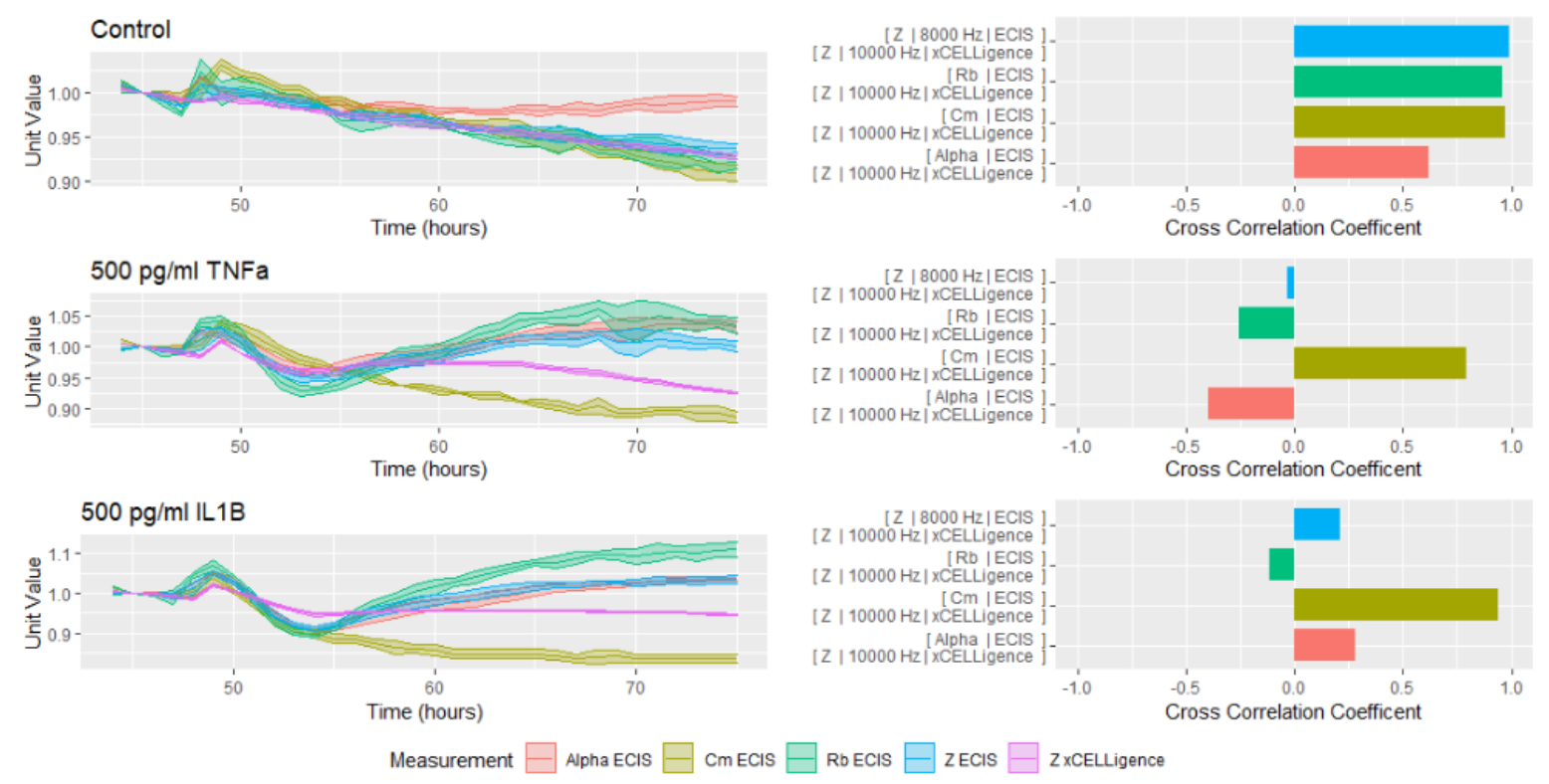

Figure 5. xCELLigence data is an inappropriate proxy for all ECIS measurements, particularly during stimulation. HCMVEC were plated at a density of 250,000 cells $/ \mathrm{cm}^{2}$ and continuously monitored for $48 \mathrm{~h}$. Cells were then treated with TNF $\alpha$, IL1 $\beta$ or vehicle and measurement was continued. Cross correlation analysis was then carried out between the average curves from all treatments for each modelled unit. Results show the mean \pm SEM from one representative experiment.

\section{Conclusions}

Although all three impedance instruments are able to detect the response of hCMVECs to inflammatory cytokines, and these are qualitatively similar in response, the sensitivities of the instruments and ability to infer cellular processes varies considerably. Furthermore, the ability to generate modelled data increases the apparent sensitivity of the measurements. This is highly relevant where a stimulus changes a single parameter (e.g., barrier strength TER vs. $R b$ ), where the modelled data will show this response clearly whereas the total resistance or total impedance may 
not. Therefore, the selection of the most appropriate impedance-sensing instrument is critical, and should be a key consideration in experimental design. Equally, the interpretation of the resultant data should be considered in the context of the threshold sensitivity of the technology.

Author Contributions: Conceptualization, J.J.W.H., C.E.A. and E.S.G.; Methodology, J.J.W.H., A.A., S.J.O., C.E.A., and E.S.G.; Software, J.J.W.H.; Formal Analysis, J.J.W.H., C.E.A., and E.S.G.; Resources, S.J.O., C.E.A., and E.S.G.; Data Curation, J.J.W.H. and E.S.G. ; Writing-Original Draft Preparation, J.J.W.H., A.A., C.E.A. and E.S.G.; Writing - Review \& Editing, J.J.W.H., A.A., S.J.O., C.E.A., and E.S.G.; Supervision, C.E.A. and E.S.G.; Project Administration, J.J.W.H., C.E.A. and E.S.G. ; Funding Acquisition, J.J.W.H., A.A., C.E.A. and E.S.G.

Funding: J.J.W.H. was supported by an AMRF Doctoral Scholarship and A.A. was supported by a Neurological Foundation Doctoral Scholarship. Instrument purchase was supported by the New Zealand Lottery Health Fund

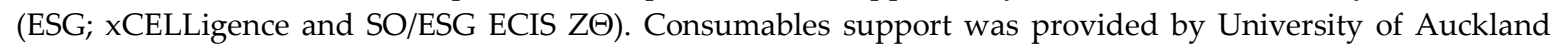
Faculty Research Development Fund (C.E.A.).

Acknowledgments: We thank the University of Auckland Statistical Consulting Centre for their input on the statistical analysis.

Conflicts of Interest: The authors declare no conflicts of interest. There was no involvement of the funders in any role pertaining to the choice of research project; design of the study; in the collection, analyses or interpretation of data; in the writing of the manuscript; or in the decision to publish the results.

\section{References}

1. Giaever, I.; Keese, C.R. Monitoring fibroblast behavior in tissue culture with an applied electric field. Proc. Natl. Acad. Sci. USA 1984, 81, 3761-3764.

2. Wegener, J.; Keese, C.R.; Giaever, I. Electric cell-substrate impedance sensing (ECIS) as a noninvasive means to monitor the kinetics of cell spreading to artificial surfaces. Exp. Cell Res. 2000, 259, 158-166.

3. Lo, C.M.; Keese, C.R.; Giaever, I. Impedance analysis of MDCK cells measured by electric cell-substrate impedance sensing. Biophys. J. 1995, 69, 2800-2807.

4. Olmo, A.; Yúfera, A. Computer simulation of microelectrode based bio-impedance measurements with comsol. In Proceedings of the BIODEVICES 2010-Proceedings of the Third International Conference on Biomedical Electronics and Devices, Valencia, Spain, 20-23 January 2010.

5. Soscia, D.A.; Lam, D.; Tooker, A.C.; Enright, H.A.; Triplett, M.; Karande, P.; Peters, S.K.G.; Sales, A.P.; Wheeler, E.K.; Fischer, N.O. A flexible 3-dimensional microelectrode array for: In vitro brain models. Lab Chip 2020, 20, 901-911.

6. Yúfera, A.; Rueda, A.; Muñoz, J.M.; Doldán, R.; Leger, G.; Rodríguez-Villegas, E.O. A tissue impedance measurement chip for myocardial ischemia detection. IEEE Trans. Circuits Syst. I Regul. Pap. 2005, 52, 26202628.

7. Pérez, P.; Huertas, G.; Maldonado-Jacobi, A.; Martín, M.; Serrano, J.A.; Olmo, A.; Daza, P.; Yúfera, A. Sensing Cell-Culture Assays with Low-Cost Circuitry. Sci. Rep. 2018, 8, 8841.

8. ECIS Z-Theta-Applied BioPhysics. Available online: https://www.biophysics.com/ztheta.php (accessed on 13 October 2020).

9. Robilliard, L.D.; Kho, D.T.; Johnson, R.H.; Anchan, A.; O'Carroll, S.J.; Graham, E.S. The importance of multifrequency impedance sensing of endothelial barrier formation using ECIS technology for the generation of a strong and durable paracellular barrier. Biosensors 2018, 8, 64 .

10. Continuously Monitor Live Cell Changes with xCELLigence RTCA SPIAgilent. Available online: https://www.agilent.com/en/product/cell-analysis/real-time-cell-analysis/rtca-analyzers/xcelligence-rtcasp-single-plate-741232\#literature (accessed on 13 October 2020).

11. cellZscope 2-nanoAnalytics Av. Available online: https://www.nanoanalytics.com/en/products/cellzscope/cellzscope2.html\#cZs_applications (accessed on 13 October 2020).

12. Bischoff, I.; Hornburger, M.C.; Mayer, B.A.; Beyerle, A.; Wegener, J.; Fürst, R. Pitfalls in assessing microvascular endothelial barrier function: Impedance-based devices versus the classic macromolecular tracer assay. Sci. Rep. 2016, 6, 1-11.

13. Wiltshire, R.; Nelson, V.; Kho, D.T.; Angel, C.E.; O'Carroll, S.J.; Graham, E.S. Regulation of human cerebromicrovascular endothelial baso-lateral adhesion and barrier function by S1P through dual involvement of S1P 1 and S1P 2 receptors. Sci. Rep. 2016, 6, 1-13. 
14. Morgan, K.; Gamal, W.; Samuel, K.; Morley, S.D.; Hayes, P.C.; Bagnaninchi, P.; Plevris, J.N. Application of Impedance-Based Techniques in Hepatology Research. J. Clin. Med. 2019, 9, 50.

15. Hillger, J.M.; Lieuw, W.L.; Heitman, L.H.; IJzerman, A.P. Label-free technology and patient cells: From early drug development to precision medicine. Drug Discov. Today 2017, 22, 1808-1815.

16. Maherally, Z.; Fillmore, H.L.; Tan, S.L.; Tan, S.F.; Jassam, S.A.; Quack, F.I.; Hatherell, K.E.; Pilkington, G.J. Real-time acquisition of transendothelial electrical resistance in an all-human, in vitro, 3-dimensional, blood-brain barrier model exemplifies tight-junction integrity. FASEB J. 2018, 32, 168-182.

17. Eigenmann, D.E.; Xue, G.; Kim, K.S.; Moses, A.V.; Hamburger, M.; Oufir, M. Comparative study of four immortalized human brain capillary endothelial cell lines, hCMEC/D3, hBMEC, TY10, and BB19, and optimization of culture conditions, for an in vitro blood-brain barrier model for drug permeability studies. Fluids Barriers CNS 2013, 10, 33.

18. O'Carroll, S.J.; Kho, D.T.; Wiltshire, R.; Nelson, V.; Rotimi, O.; Johnson, R.; Angel, C.E.; Graham, E.S. Proinflammatory TNF $\alpha$ and IL-1 $\beta$ differentially regulate the inflammatory phenotype of brain microvascular endothelial cells. J. Neuroinflammation 2015, 12, 131.

19. Kho, D.; MacDonald, C.; Johnson, R.; Unsworth, C.; O'Carroll, S.; Mez, E.; Angel, C.; Graham, E. Application of xCELLigence RTCA Biosensor Technology for Revealing the Profile and Window of Drug Responsiveness in Real Time. Biosensors 2015, 5, 199-222.

20. Venables, W.N.; Ripley, B.D. Time Series Analysis. In Modern Applied Statistics with S; Springer: Berlin/Heidelberg, Germany, 2002; pp. 387-418.

Publisher's Note: MDPI stays neutral with regard to jurisdictional claims in published maps and institutional affiliations.

(C) 2020 by the authors. Submitted for possible open access publication under the terms and conditions of the Creative Commons Attribution (CC BY) license (http://creativecommons.org/licenses/by/4.0/). 